\title{
Resonant Pedestal Pressure Reduction Induced by a Thermal Transport Enhancement due to Stochastic Magnetic Boundary Layers in High Temperature Plasmas
}

\author{
O. Schmitz, ${ }^{1}$ T. E. Evans, ${ }^{2}$ M. E. Fenstermacher, ${ }^{3}$ E. A. Unterberg, ${ }^{4}$ M. E. Austin, ${ }^{2}$ B. D. Bray, ${ }^{2}$ N. H. Brooks, ${ }^{2}$ H. Frerichs, ${ }^{1}$ \\ M. Groth, ${ }^{3}$ M. W. Jakubowski, ${ }^{5}$ C. J. Lasnier, ${ }^{3}$ M. Lehnen, ${ }^{1}$ A. W. Leonard, ${ }^{2}$ S. Mordijck, ${ }^{6}$ R. A. Moyer, ${ }^{6}$ T. H. Osborne, ${ }^{2}$ \\ D. Reiter, ${ }^{1}$ U. Samm, ${ }^{1}$ M. J. Schaffer, ${ }^{2}$ B. Unterberg, ${ }^{1}$ W. P. West, ${ }^{2}$ and the DIII-D and TEXTOR Research Teams \\ ${ }^{1}$ Forschungszentrum Jülich GmbH, IEF4-Plasma Physics, 52428 Jülich, Germany \\ ${ }^{2}$ General Atomics, San Diego, California 92186-5608, USA \\ ${ }^{3}$ Lawrence Livermore National Laboratory, Livermore, California, USA \\ ${ }^{4}$ Oak Ridge Institute for Science and Education, Oak Ridge, Tennessee, USA \\ ${ }^{5}$ Max Planck Institut für Plasmaphysik, Greifswald, Germany \\ ${ }^{6}$ University of California, San Diego, La Jolla, California 92093, USA
}

(Received 4 June 2009; published 16 October 2009)

\begin{abstract}
Good alignment of the magnetic field line pitch angle with the mode structure of an external resonant magnetic perturbation (RMP) field is shown to induce modulation of the pedestal electron pressure $p_{e}$ in high confinement high rotation plasmas at the DIII-D tokamak with a shape similar to ITER, the next step tokamak experiment. This is caused by an edge safety factor $q_{95}$ resonant enhancement of the thermal transport, while in contrast, the RMP induced particle pump out does not show a significant resonance. The measured $p_{e}$ reduction correlates to an increase in the modeled stochastic layer width during pitch angle variations matching results from resistive low rotation plasmas at the TEXTOR tokamak. These findings suggest a field line pitch angle resonant formation of a stochastic magnetic edge layer as an explanation for the $q_{95}$ resonant character of type-I edge localized mode suppression by RMPs.
\end{abstract}

DOI: 10.1103/PhysRevLett.103.165005

The impact of periodic perturbations on strongly coupled media reveal common physical features in very different physical states. In the dusty material rings around Saturn, for example, gaps are formed by gravitational resonances between the periodic motion of Saturn's moons and the strongly collisional material of the dynamic outer ring [1]. A comparable resonant mechanism is also employed for fine-tuning of the strong confining magnetic field in tokamak experiments. Here, the edge magnetic field line trajectories are perturbed by an external resonant magnetic perturbation (RMP) field with a mode structure aligned to the magnetic field line pitch angle on selected rational surfaces in the plasma edge. The periodic kicks experienced by the toroidally revolving field lines lead to the formation of an open stochastic system [2] which is a promising candidate for control of the self-organized plasma edge pedestal formed in high confinement (H-mode) plasmas [3]. In this regime, steep edge pressure gradients generate large low frequency type-I edge localized modes (ELMs) [4]. They induce transient outward heat and particle fluxes, which are expected to limit the lifetime of the divertor and first wall in the next step tomakak experiment ITER [5], potentially also degrading the plasma performance by enhanced impurity release. Therefore, the control of the ELM instabilities is a high priority physics issue for ITER. Using a periodic perturbation of the field line trajectories forming a stochastic magnetic edge layer applies a generic physics mechanism for improvement of this man-made high energy state.
PACS numbers: 28.52.-s, 52.25.Fi, 52.55.Fa, 52.55.Rk

The complete suppression of type-I ELMs by application of small edge RMP fields, having a dominant toroidal mode number $n=3$, was demonstrated at DIII-D [6] and explored for ITER similar shape (ISS) plasmas with high averaged triangularity $\bar{\delta} \sim 0.5$ at ITER-relevant, low pedestal electron collisionality $\nu_{e}^{*} \sim 0.1$ [7]. This led to a proposal for a RMP coil set for ITER [8]; for preparation of this undertaking, plans are being made to equip practically every large tokamak in the world with RMP coils. For these projects and the eventual scaling of the pioneering results from DIII-D to ITER, a basic understanding of the physical mechanisms involved is mandatory. Of major importance is the understanding of the coupling mechanism of the external field and of the eventual impact on the particle and energy transport.

In high resistivity, low rotation L-mode plasmas at the TEXTOR tokamak experiment, manifold evidence is found for a field line pitch angle resonant coupling of the external RMP field in agreement with the vacuum magnetic field modeling [9-12], i.e., the linear superposition of the external RMP field onto a stationary 2D equilibrium [2]. However, these findings were obtained at high resonant field amplitudes in the order of $10^{-3}$ relative to the confining toroidal field. Recent drift-fluid modeling of the plasma response on the external RMP field for typical TEXTOR cases [13] shows at similar high resonant field amplitudes vacuumlike penetration of the external field while at low amplitudes, a compensation of the external fields by internal response currents takes place. Also, for DIII-D H-mode plasmas with high rotation and low resis- 
tivity, a plasma response is predicted by ideal [14] and resistive [15] MHD theory. These approaches raise the question for both plasma regimes of how far the perturbed system evolving can be described at all as an open stochastic system as predicted by the vacuum field modeling. In this Letter, results of experiments are presented in which the edge safety factor $q_{95}$ was programmed to dynamically track a resonant ridge in the poloidal mode number spectrum of the external RMP field. It is shown that the alignment of the internal field line pitch angle with the external field dominates for considerably high perturbation amplitudes the plasma response in ISS plasmas at high triangularity in DIII-D. The first hint for a pitch resonance is the robust observation that for a given plasma scenario and for fixed RMP spectral properties, ELM suppression at DIII-D depends strongly on the value of the edge safety factor $q_{95}$ [6,16]. As the typical RMP spectrum used at DIII-D has dominant poloidal $(m)$ and toroidal $(n)$ mode numbers of $8<m<14$ and $n=3$, ELM suppression is observed for ISS plasmas at low $\nu_{e}^{*}$ in a narrow resonant window with a width of $\Delta q_{95}=0.1-0.48$ around $q_{95}=3.55$ [17]. In this Letter, the dynamics of this $q_{95}$ resonant feature of ELM suppression is analyzed, providing first direct evidence for a pitch resonant coupling of the external field inducing an enhanced thermal transport in contrast to previous results in low triangularity shape $[6,18]$.

In Fig. 1, an overview of the experiment is shown. Here, time traces for DIII-D discharge \#132741 with an ISS plasma at $\nu_{c}^{*} \approx 0.15$ and even parity, i.e., up-down symmetric RMP coil current distribution, are depicted. The edge safety factor $q_{95}$ was ramped down from $q_{95}=4.1$ to $q_{95}=3.2$ by ramping up the plasma current sweeping $q_{95}$ across a resonant ridge in the poloidal mode number spectrum of the RMP field. The pedestal $p_{e}(t)$ time trace and the correlation to ELM suppression are analyzed in Fig. 1, lower row, where the $p_{e}(t)$ time trace at $\Psi_{N}=0.8$ is compared to the ELM size and frequency-measured by spikes in the divertor $D_{\alpha}$-recycling emission amplitude at the inner divertor.

During $q_{95}$ ramp-down, $p_{e}(t)$ is altered with three pronounced minima at $t=3.0-3.1 \mathrm{~s}, t=3.6-3.9 \mathrm{~s}$, and $t=$ $4.35 \mathrm{~s}$, and we observe three windows in which the ELM activity is completely eliminated for $2.9 \mathrm{~s}<t_{1}<3.2 \mathrm{~s}$ interrupted by several large ELMs, $3.4 \mathrm{~s}<t_{2}<4.2 \mathrm{~s}$ and $4.45 \mathrm{~s}<t_{3}<4.7 \mathrm{~s}$. However, there is no compelling correlation found between the width of the ELM suppression windows and the maximum $p_{e}$ reduction spots. Instead, these data show that the reduction in $p_{e}$ is a prerequisite for ELM suppression; however, inside of an ELM suppressed window, the pedestal electron pressure can-depending on $q_{95}$-increase inducing new relaxation processes. Also, comparison of the absolute $p_{e}$ value in the first (e.g., at $t=$ $3.0 \mathrm{~s}$ ), second (at $t=3.7 \mathrm{~s}$ ), and third (at $t=4.6 \mathrm{~s}$ ) suppression window shows the important fact that while the actual $p_{e}$ value is not directly correlated to ELM suppression, ELMs can be suppressed with a moderate reduction of the pedestal $p_{e}$.

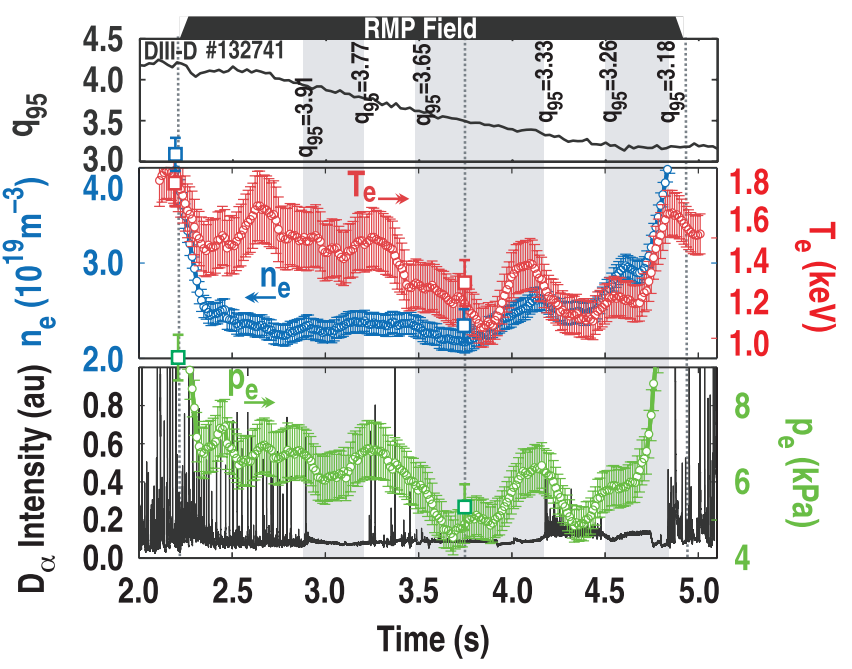

FIG. 1 (color). Pedestal time traces in DIII-D discharge \#132741 with $q_{95}$ ramp-down (from top to bottom): $q_{95}(t)$, $T_{e}(t)$, and $n_{e}(t)$ and the resulting $p_{e}(t)$ at $\Psi_{N}=0.8$ including the $D_{\alpha}$-recycling emission light at the inner divertor strike line. ELM suppressed periods are marked with grey shaded boxes and the RMP period is indicated on top. The square markers show $n_{e}$, $T_{e}$, and $p_{e}$ values for a second discharge at fixed $q_{95}=3.5$. The error bars shown are the fitting and statistical measurement uncertainties.

This $q_{95}$ dependence of the pedestal $p_{e}(t)$ is the result of a very different manipulation of the pedestal (at $\Psi_{N}=0.8$ ) electron density $n_{e}(t)$ and temperature $T_{e}(t)$ time traces shown in Fig. 1, middle row. A density pump out [19] is seen as an overall reduction of $n_{e}(t)$ as soon as the RMP field is applied reducing the ELM size and increasing the ELM frequency. No significant $q_{95}$ dependency of this density pump out is seen, and in contrast, $T_{e}(t)$ is highly $q_{95}$ dependent. At the start of the $q_{95}$ ramp shown, only a small $T_{e}$ reduction occurs followed by a localized increase of $T_{e}$ at $t=2.6 \mathrm{~s}$. This keeps the plasma pressure on a level where no ELM suppression is established yet. As soon as $T_{e}(t)$ decreases slightly, $p_{e}(t)$ also decreases and the first ELM suppression window appears. From this time on, $T_{e}(t)$ decreases nearly monotonically until $t=3.9 \mathrm{~s}$ when it increases correlated with $n_{e}(t)$ building up $p_{e}(t)$ to a level where the small $D_{\alpha}$ bursts indicate a marginally stable situation. This observation shows for the first time that the $q_{95}$ dependent alteration of $T_{e}(t)$, i.e., a $q_{95}$ resonant manipulation of the thermal transport combined with an overall density pump out, causes the $q_{95}$ resonant nature of ELM suppression in ISS plasmas at high triangularity. As the pressure gradient drives the peeling-ballooning theory of the ELM evolution [4], a reduction of the total pressure $p\left(\Psi_{N}\right)$ and $\nabla_{p}\left(\Psi_{N}\right)$ is discussed as an underlying mechanism for ELM suppression by RMP [6,20]. This new finding was confirmed for comparable discharges at fixed $q_{95}=3.5$, i.e., the most reliable ELM suppression working point. As the actual values of $T_{e}, n_{e}$, and $p_{e}$ depend strongly on wall condition [19], collisionality [16], and plasma shape [7], the results are taken from discharge 
\#132731 of the same day with the same shape and the same $\nu_{e}^{*} \sim 0.15$. The values of $n_{e}, T_{e}$, and $p_{e}$ for fixed $q_{95}$ are included in Fig. 1, middle and lower rows, as square markers with corresponding colors. This proves that the same relative change of the pedestal values with and without RMP field observed during $q_{95}$ ramping can be reliably reproduced at fixed $q_{95}=3.5$.

This strong $T_{e}\left(q_{95}\right)$ dependency was analyzed in more detail comparing the electron temperature time traces obtained at $\Psi_{N}=0.7$ with the electron cyclotron emission (ECE) system and with the Thomson scattering diagnostic as shown in Fig. 2. The ECE data resemble directly the $T_{e}\left(q_{95}\right)$ modulation measured by Thomson scattering. Moreover, this direct comparison reveals that the correlated $T_{e}$ reaction occurs for the ECE data systematically at slightly lower $q_{95}$ values, i.e., later in time than for the Thomson scattering data. As both diagnostics measure at different toroidal and poloidal locations, this fact provides further evidence for the evolution a stochastic boundary with a three-dimensional topology as shown experimentally in Refs. [21,22] and studied numerically in detail in Ref. [23]. Additionally, the square markers show values of discharge \#132731 at fixed $q_{95}=3.5$. They confirm that the decrease in $T_{e}$ between the RMP and no-RMP phases are practically the same for stationary $q_{95}$ and during $q_{95}$ ramping. This reduction in the pedestal $T_{e}$ values is a feature seen in ITER similar shaped plasmas at high triangularity, which contrasts results of plasmas at low triangularity, where the thermal transport was only weakly affected $[6,18]$.

In order to elucidate the radial behavior of the characteristic pressure modulation, we display in Fig. 3 the $p_{e}\left(\Psi_{N}\right)$ profiles in the plasma edge region as a function of $q_{95}$ and compare this to a similar analysis from TEXTOR. In general, a pronounced modulation of $p_{e}$ with dependence on $q_{95}$ or the edge safety factor $q_{a}$ for the limiter case was observed in both experiments. For TEXTOR, the $p_{e}\left(\Psi_{N}, t\right)$ was measured by means of an ECE system $\left(T_{e}\right)$ and a laser interferometer for $\left(n_{e}\right)$ in $m / n=12 / 4$ base mode [24],

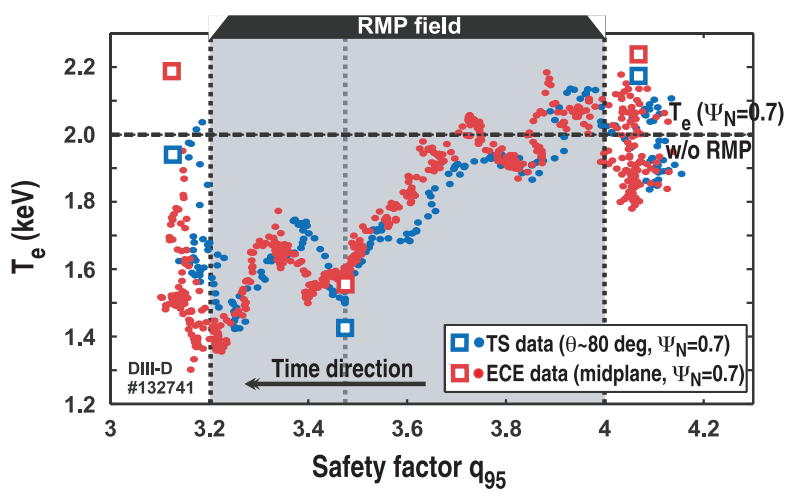

FIG. 2 (color). Comparison of $T_{e}(t)$ at $\Psi_{N}=0.7$ obtained from electron cyclotron (red dots) and Thomson scattering measurement (blue dots) during $q_{95}$ ramp-down. The square markers show the $T_{e}$ values with and without RMP for stationary $q_{95}$. similar to the RMP spectrum considered for ITER. Both experiments exhibit the most pronounced impact on $p_{e}$ in the plasma edge region, with strong $p_{e}\left(q_{95}\right)$ reduction at certain $q_{95}$ values. This $p_{e}\left(q_{95}\right)$ dependence is induced in both experiments by a resonant impact on $T_{e}$ and it is important to note that for DIII-D, the strongest $p_{e}$ reduction is found within the most robust ELM suppression window around $q_{95} \approx 3.5$.

To provide an ansatz for explanation of this strong $q_{95}$ resonance of the pedestal and edge $p_{e}(t)$ values and the underlying $T_{e}$ reduction, we analyze the perturbed field line topology for both experiments in the vacuum paradigm. In Fig. 3(b), the extension of a laminar and a stochastic zone as the two characteristic domains in the perturbed boundary of TEXTOR are depicted as function of $q_{a}$. For separation of these two domains, we compare the field line connection length $L_{c}$ with both (a) the mean free collisional path $\lambda_{c}$ of the electrons as the transport length scale and (b) the Kolmogorov length $L_{K}$ as the topological length scale, describing the correlation length of neighboring field lines [25]. The laminar field lines fulfill $L_{c}<L_{K}$ while the stochastic field lines yield $L_{c}>L_{K}$ experiencing uncorrelated stochastic trajectories [26,27]. In comparison, we calculate $\lambda_{c}$ as the transport length scale at two radial locations, one right at the separatrix or the last closed flux surface ( $\left.\lambda_{c \text { sep }}\right)$ and the other at $\Psi_{N} \sim 0.8$, i.e., on top of the

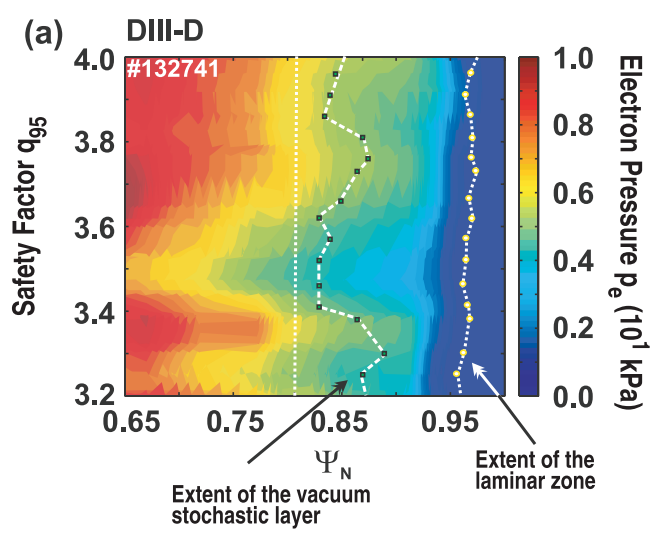

(b) TEXTOR-DED

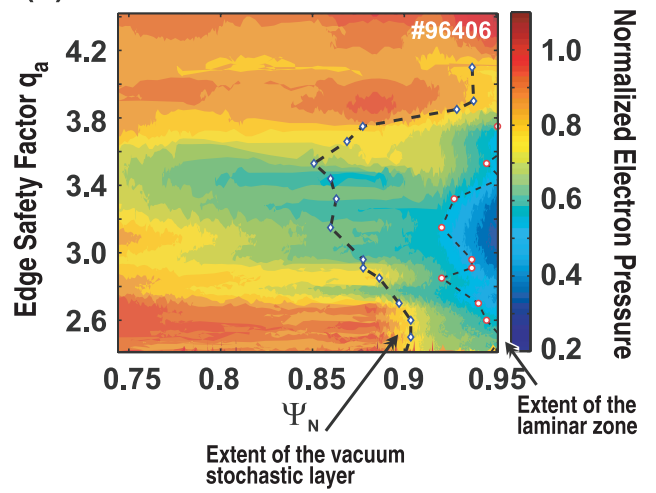

FIG. 3 (color). Modulation of electron pressure $p_{e}$ during ramp-down of the safety factor (a) at DIII-D and (b) in TEXTOR. 
pedestal for the H-mode plasma $\left(\lambda_{c \text { ped }}\right)$. For the TEXTOR case shown, we get $\lambda_{c \text { sep }} \sim 25 \mathrm{~m}$ and $\lambda_{c \text { ped }} \approx 130 \mathrm{~m} \mathrm{[10].}$ As the laminar field lines typically have $L_{c} \approx 30 \mathrm{~m}$, they yield $L_{c} \approx \lambda_{c \text { sep }}$, therefore acting as a 3D scrape-off layer due to a correlated, parallel transport to the wall $[10,28]$. Accordingly, at TEXTOR, a strong $p_{e}$ reduction dependent on the laminar layer width is seen in Fig. 3(b). However, the stochastic field lines connect as well within $L_{c} \lesssim \lambda_{c \text { ped }}$ to the wall. Therefore, the parallel particle flows and heat conduction along these field lines lead, in combination with the stochastic field line motion, to an enhanced net outward transport of particle and energy. As Fig. 3(b) shows, these field lines penetrate depending on the actual $q_{a}$ value as deep as $\Psi_{N}=0.85$, and the $p_{e}$ profile drop is strongest for maximum stochastic layer width. Accordingly, the pressure drop vanishes as soon as this layer shrinks for $q_{a}>3.8$. The comparable analysis for the DIII-D case is shown in Fig. 3(a). We obtain $\lambda_{c \text { sep }} \approx 150 \mathrm{~m}$ at the separatrix, which is similar to $L_{K}$ and $\lambda_{c \text { ped }} \approx 1000 \mathrm{~m}$ at $\Psi_{N}=$ 0.8 . The width of the laminar and stochastic zone is overlaid in Fig. 3(a). The extent of the stochastic layer resembles the measured $p_{e}$ modulation-all $p_{e}$ dips are correlated with an increased stochastic layer width while the pressure profiles recover with decreasing layer width.

In summary, a strong dependence of the edge electron pressure $p_{e}$ on the edge safety factor $q_{95}$ during RMP application was found for the two tokamak experiments DIII-D and TEXTOR with strongly different plasma regimes. This provides direct experimental evidence that on both experiments a field line pitch angle resonant coupling of the external field to the internal magnetic field line structure induces an open stochastic system which leads to a pedestal $p_{e}$ reduction due to an enhancement of the electron thermal transport. This mechanism is a prerequisite for stabilization of large type-I ELMs in DIII-D H-mode plasmas. Here, the $p_{e}\left(q_{95}\right)$ resonance is driven by a strong $T_{e}\left(q_{95}\right)$ dependence while in contrast, $n_{e}\left(q_{95}\right)$ does not show a significant $q_{95}$ resonance. A pitch angle resonant extension of the vacuum stochastic layer width correlated to the reduction of $T_{e}$ is seen, providing evidence that heat conduction along the stochastic field lines-and therefore enhanced outward heat transport-can explain the $q_{95}$ resonant reduction in $T_{e}$ on both experiments. This also agrees qualitatively for the first time in an $\mathrm{H}$-mode plasma with predictions by analytical models for heat transport in a stochastic boundary [29] and with numerical 3D fluid modeling for DIII-D [30]. This enhancement in the electron thermal transport for RMP ELM suppressed H-mode plasmas at DIII-D in high triangularity plasmas contrasts previous experimental results in low triangularity shape [6], revealing a strong shape dependence of the thermal transport.

A direct correlation of the radial extent of the $p_{e}$ reduction zone shown in Fig. 3 to the stochastic layer extension is difficult to extract as the DIII-D H-mode profiles are stiff due to the internal transport barrier compared to the volatile TEXTOR L-mode profiles. Therefore, the wide radial extent of the $p_{e}$ reduction for the DIII-D case, compared to an extension following very closely the modeled stochastic layer width for the TEXTOR case, does not directly imply a difference in the field penetration. Determining the actual local perturbation amplitude inside of the plasma and the resulting complete perturbed field topology including a plasma response is a challenge for future studies. However, the results presented in this Letter provide substantial evidence that the basic effect, which drives the field line pitch resonant characteristics of ELM suppression and the underlying profile modifications, compares well to the generic physics picture of formation of an open stochastic system.

This work was supported in part by the U.S. Department of Energy under Grants No. DE-FC02-04ER54698, No. DE-AC52-07NA27344, No. DE-AC05-06ER23100, and No. DE-FG02-07ER54917.

[1] D. P. Hamilton et al., Nature Phys. 1, 87 (2005).

[2] T. E. Evans et al., Phys. Plasmas 9, 4957 (2002).

[3] F. Wagner et al., Plasma Phys. Controlled Fusion 49, B1 (2007).

[4] P. B. Snyder et al., Nucl. Fusion 44, 320 (2004).

[5] A. Loarte et al., Nucl. Fusion 47, S203 (2007).

[6] T. E. Evans et al., Nature Phys. 2, 419 (2006).

[7] T. E. Evans, Nucl. Fusion 48, 024002 (2008).

[8] M. J. Schaffer et al., Nucl. Fusion 48, 024004 (2008).

[9] M. Jakubowski et al., Phys. Rev. Lett. 96, 035004 (2006).

[10] O. Schmitz et al., Nucl. Fusion 48, 024009 (2008).

[11] M. Jakubowski et al., J. Nucl. Mater. 363-365, 371 (2007).

[12] A. Wingen et al., Phys. Plasmas 14, 042502 (2007).

[13] D. Reiser et al., Phys. Plasmas 16, 042317 (2009).

[14] J. K. Park et al., Phys. Rev. Lett. 99, 195003 (2007).

[15] V. Izzo et al., Nucl. Fusion 48, 115004 (2008).

[16] M.E. Fenstermacher et al., Phys. Plasmas 15, 056122 (2008).

[17] T. E. Evans et al., Proceedings of the 2008 IAEA Conference, Geneva, Vol. EX4/1, available at http://www-pub. iaea.org/MTCD/Meetings/FEC2008/ex_4-1.pdf.

[18] M. Z. Tokar et al., Phys. Rev. Lett. 98, 095001 (2007).

[19] E. A. Unterberg et al., Nucl. Fusion 49, 092001 (2009).

[20] T.H. Osborne et al., Proceedings of the 32nd EPS Conference on Plasma Physics D (European Physical Society, Mulhouse, 2005), Vol. 32, p. 4.012.

[21] T. E. Evans et al., J. Phys. Conf. Ser. 7, 174 (2005).

[22] O. Schmitz et al., Plasma Phys. Controlled Fusion 50, 124029 (2008).

[23] H. Frerichs et al., "Three-Dimensional Edge Transport Simulations for DIII-D Plasmas with Resonant Magnetic Perturbations," Nucl. Fusion (to be published).

[24] K. H. Finken et al., Nucl. Fusion 47, 522 (2007).

[25] S. S. Abdullaev et al., Phys. Plasmas 15, 042508 (2008).

[26] F. Nguyen et al., Nucl. Fusion 37, 743 (1997).

[27] P. Ghendrih et al., Nucl. Fusion 42, 1221 (2002).

[28] T. Eich et al., Nucl. Fusion 40, 1757 (2000).

[29] A. B. Rechester et al., Phys. Rev. Lett. 40, 38 (1978).

[30] I. Joseph et al., Nucl. Fusion 48, 045009 (2008). 